\title{
HIPERPLASIA ENDOMETRIAL: ANÁLISIS DE SERIE DE CASOS DIAGNOSTICADOS EN BIOPSIA ENDOMETRIAL
}

\author{
Ernesto García Ayala ${ }^{1}$, Laura Cárdenas Mastrascusa ${ }^{2}$, Diana Sandoval Martínez ${ }^{2}$, \\ Henry Mayorga Anaya. ${ }^{a}$ \\ 1 Departamento de Patología, ${ }^{2}$ Residentes Programa de Patología, Departamento de Patología, Facultad de Salud, \\ Universidad Industrial de Santander (Bucaramanga-Colombia).
}

a Alumno, Facultad de Medicina, Universidad Industrial de Santander (Bucaramanga-Colombia).

\section{RESUMEN}

Antecedentes: La hiperplasia endometrial es una entidad en la que existe una proliferación de glándulas endometriales de tamaño y forma irregular, con mayor proporción de glándulas sobre el estroma, a consecuencia de una excesiva exposición a los estrógenos. Aproximadamente, en el 15\% de legrados/biopsias endometriales de mujeres postmenopausicas con cuadro clínico de hemorragia uterina anormal, se diagnostica esta entidad. Objetivo: Describir la incidencia y hallazgos histopatológicos en legrado/biopsia endometrial en pacientes de un hospital público de tercer nivel. Métodos: Se revisaron 22.048 procedimientos realizados en el Hospital Universitario de Santander, procesados en el Departamento de Patología de la Universidad Industrial de Santander, en el periodo comprendido entre 1 de enero de 2005 y 31 de diciembre 2008 , de los cuales 1.750 correspondieron a legrados/biopsias de endometrio y en 168 de estos se realizó el diagnóstico histopatológico de hiperplasia endometrial. Resultados: Se encontró que el promedio de edad de presentación de está entidad fue de 44,8 años y que el mayor porcentaje de pacientes $(68,5 \%)$ estuvieron en el grupo de la hiperplasia simple sin atipia. En el 19,7\% de los casos hubo evidencia de atipia. Conclusiones: El promedio de edad encontrado y los porcentajes por subgrupos de hiperplasia estuvieron en relación a otros estudios. Se destaca una menor proporción de casos con atipia.

\section{PALABRAS CLAVE: Hiperplasia, biopsia de endometrio, atipia}

\section{SUMMARY}

Background: Endometrial hyperplasia is an entity in which there is a proliferation of endometrial glands of irregular size and shape, with the highest proportion of glands on the stroma, resulting from excessive exposure to estrogen. Approximately $15 \%$ of curettages/endometrial biopsies of postmenopausal women with clinical symptoms of abnormal uterine bleeding is diagnosed this entity. Objective: To describe the incidence and pathological findings in curettage/endometrial biopsy in patients of a tertiary public hospital. Methods: A retrospective review of 22,048 surgical procedures performed in the University Hospital of Santander, processed in the Pathology Department of Industrial University of Santander in the period from 1 January 2005 and 31 December 2008, of which 1,750 corresponded to curettage/biopsy of the endometrium and in 168 of these histopathological diagnosis was made of endometrial hyperplasia. Results: We found that the average age of presentation in this institution was 44.8 years and that the greater percentage of patients $(68.5 \%)$ were in the group of simple hyperplasia without atypia. In $19.7 \%$ of the cases had evidence of atypia. Conclusions: The mean age and percentages found by hyperplasia subgroups were relatively within limits with regard to other studies, although broadly outlined in a lower proportion of cases with atypia.

KEY WORDS: Hyperplasia, endometrial biopsy, atypia 


\section{INTRODUCCIÓN}

La hiperplasia endometrial es una proliferación de glándulas endometriales de tamaño y forma irregular, con un aumento de la razón glándula/estroma (1), que se desarrolla a consecuencia de una excesiva exposición a los estrógenos, sin que exista oposición a su efecto proliferativo, tal y como ocurre en los ciclos anovulatorios, o cuando se administran en terapia exógena (tratamiento de reposición estrogénica) $(1,2)$, o en un aumento de la producción de los mismos en entidades clínicas como tumores ováricos funcionantes de células de la granulosa, síndrome de ovario poliquístico y tecoma ovárico $(1,2)$.

En la actualidad son conocidas más de veinte clasificaciones de hiperplasia endometrial que intentan evaluar y establecer una correspondencia entre la morfología de la lesión y su capacidad de progresión. La Organización Mundial de la Salud (OMS) propuso inicialmente clasificarlas en hiperplasia glandular quística, adenomatosa y atípica, pero en 1992 la International Society of Gynecological Pathologist y el comité de tumores endometriales de la OMS (3), deciden adoptar la clasificación de hiperplasia simple, compleja y atípica, con base en la apariencia, aglomeración y la presencia de atípia en el epitelio glandular, como originalmente fue definida por Kurman y cols $(4,5)$, en 1985.

Marco teórico. Con base en lo anteriormente expuesto se procede a describir a continuación las características más importantes de los diferentes tipos de hiperplasias. La simple (también conocida como hiperplasia quística o leve) es una lesión proliferativa que se caracteriza por cambios arquitectónicos en las glándulas de diversos tamaños, con mínimos cambios en la complejidad y densidad glandular y abundante estroma entre las mismas. El epitelio superficial es pseudoestratificado con ocasionales figuras mitóticas y ausencia de atipia nuclear (4-9). La hiperplasia endometrial compleja es también una lesión proliferativa, en la cual se exhibe un incremento en el número y tamaño de las glándulas endometriales, que lucen apiñadas de forma irregular y con mínimo estroma interpuesto adoptando una morfología característica conocida como patrón de "espalda contra espalda" $(4,6,9,10,11)$ (Figura 1).

Basándose en la características citológicas, las hiperplasias anteriormente descritas, se subclasifican de una manera mucho más simple: hiperplasia con o sin atipia $(4,6,9,10,11)$. Dicha atipia citológica está caracterizada por un aumento en la estratificación con dispolaridad celular, núcleos hipercromáticos, núcleolo prominente, cromatina de aspecto grumoso e incremento de la razón núcleo/citoplas- ma $(5,6,10)$ (Figura 2). La presencia de atipia celular es el factor pronóstico más importante para la progresión a carcinoma endometrial. En un estudio, se encontró que el $23 \%$ de las pacientes con hiperplasias atípicas desarrollan adenocarcinoma de endometrio en un promedio de 4 años posterior al diagnóstico de la lesión precursora, mientras que solo el $2 \%$ de lesiones sin atipia progresan en un periodo estimado de 13,4 años $(5,7,11,12)$.

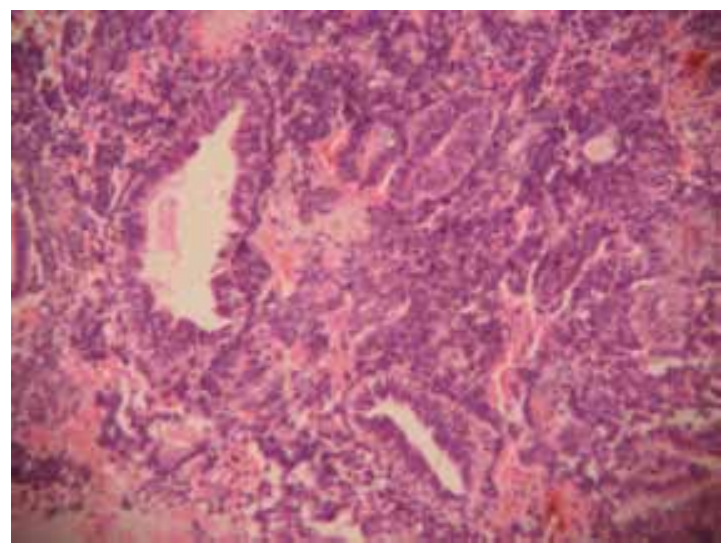

Figura1. Hiperplasia compleja con atipia. 10X (hematoxilina-eosina).

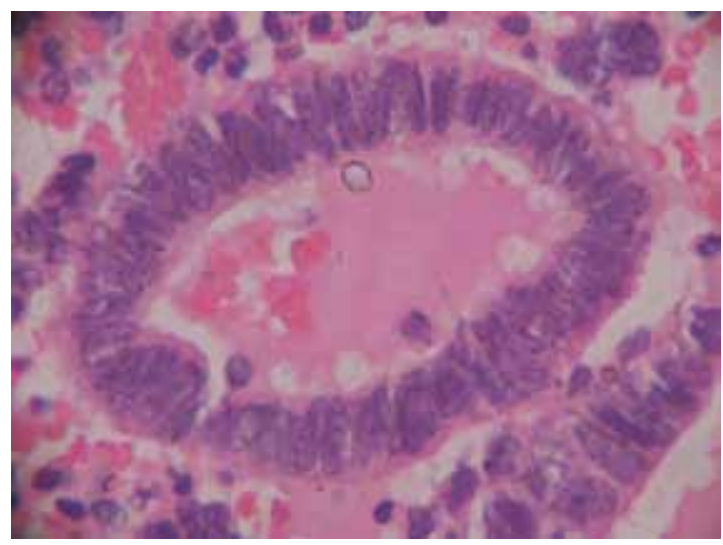

Figura 2. Atipia celular con pérdida de la polaridad, pleomorfismo e hipercromatismo nuclear. 40X (hematoxilina-eosina).

La hiperplasia endometrial atípica es una entidad histopatológica que entraña dificultades diagnósticas y terapéuticas, dada la frecuencia con que las pacientes con ese diagnóstico presentan finalmente adenocarcinoma de endometrio en la pieza de histerectomía (13). Consecuentemente un grupo de expertos que participaron en 1999 en un estudio multicéntrico europeo (14) para el análisis de 56 muestras 
de endometrio, planteó la escasa reproducibilidad intra e interobservador en el diagnóstico de la hiperplasia endometrial con esta clasificación consiguiendo mejores resultados cuando se agrupaban en tres lesiones: endometrio cíclico (proliferativo, secretor y otros) hiperplasia y neoplasia endometrial (incluye hiperplasia atípica y el adenocarcinoma endometrial bien diferenciado).

Posteriormente el Endometrial Collaborative Group (14) propuso otra clasificación: hiperplasia, neoplasia intraepitelial endometrial (NIE) y adenocarcinoma, que ha demostrado mejores resultados en porcentajes de reproducibilidad que la clasificación de la OMS (9).

Dadas las dificultades existentes para la categorización de la hiperplasia endometrial atípica o el adenocarcinoma, algunos autores han creado el concepto de neoplasia endometrial intraepitelial, que clasifica aparentemente de manera más eficaz a las pacientes con riesgo alto y bajo de cáncer y con mejor reproducibilidad que la hiperplasia endometrial atípica $(9,15,16,17)$. Sin embargo, la OMS $(5,6)$ aún mantiene la clasificación con base en la histopatología de presencia o no de atipia celular, que se resume en la Tabla I. Dicha clasificación es la que actualmente la mayoría de patólogos aún consideran describiendo estas lesiones intraepiteliales dentro del grupo de las hiperplasias atípicas $(5,6,8,9,10,11,18,19)$.

Diversos factores se han descrito en el aumento de lesiones precursoras de endometrio, al igual que del adenocarcinoma endometrial. La terapia de reemplazo hormonal al parecer es el factor de riesgo más importante para el desarrollo de hiperplasia endometrial. En el estudio PEPI (20) (Postmenopausal Estrogen/Progestin Interventions Trial) quedó claramente demostrado el riesgo de los estrógenos sin progestágenos al igual que en otros estudios (21). Durante tres años de tratamiento estrogénico continuado, el $62 \%$ de las mujeres desarrollaron una hiperplasia frente a sólo el $2 \%$ de las que se les administró placebo (22). Unfer y cols (23), reportan un leve aumento de la incidencia de hiperplasia

\section{Tabla I}

\section{CLASIFICACIÓN HISTOPATOLÓGICA DE LA HIPERPLASIA ENDOMETRIAL (6)}

$\begin{array}{ll}\begin{array}{l}\text { Hiperplasia endometrial } \\ \text { sin atipia } \\ \text { (Bajo Grado) }\end{array} & \begin{array}{l}\text { Simple } \\ \text { Compleja }\end{array} \\ \begin{array}{l}\text { Hiperplasia endometrial } \\ \text { con atipia }\end{array} & \text { Simple } \\ \text { (Alto Grado) } & \text { Compleja }\end{array}$

endometrial en pacientes con terapia hormonal con fitoestrógenos.

La obesidad, es un problema de salud pública en el mundo occidental que se asocia a un incremento de hasta 5 veces más riesgo de padecer una neoplasia endometrial (24). En mujeres premenopáusicas, la obesidad y el síndrome de ovario poliquístico están asociados a ciclos anovulatorios, y como consecuencia de ello, el endometrio se encuentra expuesto a la acción de los estrógenos en ausencia de la acción reguladora de la progesterona. En la posmenopausia, la obesidad conlleva a exposición del endometrio a cantidades elevadas de estrógenos provenientes de la trasformación periférica de la androstendiona (A4) a estrona (E1) y estradiol (E2), los cuales en ausencia del efecto regulador de la progesterona, estimulan la proliferación endometrial que puede progresar a hiperplasia endometrial (25). Ricci y cols (26), en un estudio de casos y controles, establecieron el riesgo (Odds Ratios) para diferentes factores evaluados en su estudio, los cuales se resumen en la Tabla II, además encontraron que no existía una asociación significativa con la historia familiar de cáncer de endometrio, ovario o mama (26). Los tumores de ovario de células de la granulosa y tecomas así como algunos tumores de la corteza suprarrenal, son entidades poco frecuentes en la génesis de hiperplasia endometrial $(5,12)$.

Actualmente los factores genéticos cobran mayor importancia en el desarrollo de la hiperplasia endometrial y los adenocarcinomas relacionados. Es así, como la inactivación del gen supresor de tumores PTEN (phosphatase and tensin homolog deleted on chromosome 10$)(5,9,16,27-30)$, el cual codifica una fosfatasa, facilita la remoción de grupos fosfatos de moléculas Akt y mTOR quinasas, que se encuentran en la vía intracelular de la fosfoinositol 3-quinasa (PI3K) $(9,16,30,31)$. La actividad de PTEN produce una detención del ciclo celular y

Tabla II

\section{FACTORES DE RIESGO PARA DESARROLLO DE HIPERPLASIA ENDOMETRIAL (26)}

\begin{tabular}{lc}
\hline FACTOR DE RIESGO & OR (IC95\%) \\
\hline Terapia de reemplazo hormonal & $3,1(1,1-9,3)$ \\
Obesidad & $2,7(1,5-5,0)$ \\
Diabetes & $2,5(0,8-6,9)$ \\
Edad de menopausia > 53 años & $2,2(0,7-6,7)$ \\
Paridad > 2 hijos & $1,8(0,9-3,6)$ \\
$\begin{array}{l}\text { Uso de anticonceptivos } \\
\text { orales (acos) }\end{array}$ & $1,6(0,9-2,8)$ \\
\hline
\end{tabular}


la apoptosis, así como la inhibición de la motilidad celular. Por lo tanto, con la pérdida de su expresión, las células ingresan en el ciclo celular y generan una excesiva población celular, se ha demostrado que esta deleción es un evento temprano en la carcinogénesis endometrial $(5,13,27,28)$ que favorece además que las células se vuelvan más sensibles a la estimulación estrogénica $(5,8,13,27,28,30)$.

El oncogén bcl-2 $(27,29,30)$, localizado en el cromosoma 18 y sujeto a regulación por control hormonal ha sido descrito en la patogénesis de la hiperplasia endometrial. Un aumento de su expresión en la fase proliferativa y su marcado descenso en la fase secretora ha sugerido un papel central en la inhibición de la apoptosis, prolongando la sobrevida celular. El incremento sustancial de la expresión del bcl-2 ha quedado completamente demostrado en la hiperplasia endometrial compleja $(7,29)$ y se ha propuesto una interacción entre la expresión de este oncogén y el gen Fas/Fasl podría ser uno de los factores que contribuya en el desarrollo de la hiperplasia endometrial $(7,32)$.

Otros autores sugieren que la presencia de inestabilidad de microsatélites, mutaciones en el oncogén K-ras $(10,33)$, y en el gen CTNNB1 se encuentran presentes en lesiones precursoras de adenocarcinoma de endometrio (34).

La principal manifestación clínica de la hiperplasia endometrial es la hemorragia uterina anormal, y aproximadamente en un $15 \%$ de pacientes con historia de hemorragia uterina anormal, se hace diagnóstico de alguna forma de hiperplasia $(7,35)$ por lo cual conlleva a hacer diagnósticos diferenciales de acuerdo al grupo etario de la paciente (Tabla III) $(5,7)$.

La relación de la hiperplasia endometrial y el adenocarcinoma de endometrio ha quedado de- mostrada: la mayoría de los adenocarcinomas tienen hiperplasia de endometrio previas entre un 33 y $75 \%$ de los casos $(9,19)$. En un estudio clásico de Kurman y cols (7), el $2 \%$ de las pacientes con diagnóstico inicial de hiperplasia sin atipia progresan a carcinoma, el $23 \%$ de las pacientes con diagnóstico de hiperplasia (simple y compleja) con atipia progresan a adenocarcinoma. Dentro de las pacientes con diagnóstico de hiperplasia compleja con atipia el $29 \%$ de ellas progresan en último término a adenocarcinoma endometrial. Sin embargo en una reciente publicación la evolución de la hiperplasia atípica a carcinoma ocurrió en el $52 \%$ de los casos; cuando el estudio histopatológico del espécimen de biopsia endometrial diagnostica hiperplasia atípica, el riesgo de carcinoma concomitante ha sido reportado entre el 17 y el $25 \%$ de las veces (7); sin embargo en otras publicaciones este porcentaje asciende hasta un $40-42 \%$ (34).

El objetivo de esta comunicación es describir la incidencia y hallazgos histopatológicos en legrado/ biopsia endometrial en pacientes de un hospital público de tercer nivel.

\section{PACIENTES Y METODOS}

Estudio descriptivo retrospectivo de una serie de casos en biopsias endometriales de pacientes del Hospital Universitario de Santander (HUS), procesadas y evaluadas en el Departamento de Patología de la Universidad Industrial de Santander(UIS) de la ciudad de Bucaramanga-Colombia, en el periodo comprendido entre el 1 de enero de $2005 \mathrm{y} \mathrm{el}$ 31 de diciembre de 2008.

Se revisaron un total de 22.048 informes histopatológicos de los cuales 1.750 correspondieron a

Tabla III

\section{CAUSAS DE HEMORRAGIA UTERINA ANORMAL SEGÚN EL GRUPO ETARIO}

\begin{tabular}{ll}
\hline & Causas \\
\hline Prepúberes & Pubertad precoz (origen ovárico, hipofisiario o hipotalámico) \\
Adolescencia & Ciclos anovulatorios, desórdenes de la coagulación \\
Edad reproductiva & Complicaciones obstétricas (aborto, embarazo ectópico, \\
& enfermedad trofoblástica gestacional) \\
& Lesiones orgánicas (leiomiomas, adenomiosis, hiperplasia endometrial) \\
& Ciclos anovulatorios \\
Sangrado disfuncional ovárico (fase lútea inadecuada) & Ciclos anovulatorios \\
Perimenopausia & Lesiones orgánicas (carcinoma, hiperplasia, pólipos) \\
Postmenopausia & Lesiones orgánicas (carcinoma, hiperplasia, pólipos)
\end{tabular}


legrados/biopsias de endometrio y en 168 de estos se confirmó el diagnóstico histopatológico de algún tipo de hiperplasia endometrial. Se evaluó la edad promedio en el momento del diagnóstico, así como también la incidencia por subgrupo de clasificación histopatológica y el rango de edad más frecuente en el cual se presentó esta entidad clínica. Para la discusión de los datos obtenidos se realizó una búsqueda de publicaciones en la base de datos de PubMed.

\section{RESULTADOS}

De los 1.750 legrados uterinos, 168 casos correspondieron al diagnóstico histopatológico de hiperplasia endometrial, lo cual indica que en el 9,6\% de total de legrados/biopsias de endometrio realizados en el HUS, se diagnosticó esta entidad. El promedio de edad al momento del diagnóstico fue de 44,8 años, el rango más frecuente fue el comprendido entre los 41 y 50 años $(61,32 \%)$ y el de menor frecuencia en mujeres mayores de 60 años $(1,79 \%)$.

La variante histopatológica más frecuente fue la hiperplasia endometrial simple sin atipia, que se observó en $115(68,5 \%)$ de los 168 casos analizados, con una edad promedio de 45,5 años. En contraparte, 7 casos $(4,2 \%)$ tuvieron el diagnóstico de hiperplasia simple con atipia, constituyéndose en el subgrupo con menor frecuencia diagnóstica, con una edad promedio de 44,3 años.

La hiperplasia compleja con atipia, es la segunda en orden de frecuencia, observándose en 26 casos (15,5\%). Este subgrupo histológico presentó la mayor edad promedio (46,7 años). La hiperplasia compleja sin atipia, se diagnosticó en 20 casos $(11,9 \%)$. La edad promedio fue la menor encontrada en esta casuística (42,7 años) (Tabla IV).

El rango de edad que presenta mayor frecuencia de casos está entre los 41 y 50 años, con
103 del total de los 168 analizados, que equivale al $61,3 \%$. Se destaca que en todos los subgrupos histológicos de hiperplasia endometrial el mayor número de pacientes se ubicó igualmente en el anterior grupo etario. En segundo lugar está el grupo de edad comprendido entre los 31 y 40 años, con 29 pacientes (17,3\%). Entre los 51 y 60 años, se diagnosticaron 27 casos, lo cual equivale al 16,1\%. Sin embargo este rango de edad es el segundo en frecuencia en el subgrupo de las hiperplasias complejas con atipia. Por otro lado, solo hubo 3 pacientes mayores de 60 años en quienes se hizo este diagnóstico (1,8\%). En el grupo de pacientes con edades menores a 30 años, se diagnosticaron 6 casos lo que representa un 3,6\%, y es el doble de casos diagnosticados en pacientes mayores de 60 años, empero, ninguno de estos dos grupos presentó casos de hiperplasia con atipia (Tabla V).

\section{DISCUSIÓN}

Los datos del estudio de Registro Poblacional de Cáncer del Área Metropolitana de Bucaramanga (Santander/Colombia) (36) entre los años 2000 a 2004, mostraron que las lesiones tumorales malignas del cuerpo del útero representaron el 3,7\% de todas las neoplasias malignas de la mujer. Se captaron un total de 123 casos de tumor invasivo de esta estructura de los cuales 113 casos $(91,9 \%)$ afectaron al endometrio, siendo fundamentalmente en su gran mayoría adenocarcinomas de tipo endometroide de diferentes grados de diferenciación. Si se tiene en cuenta la secuencia y progresión establecida entre hiperplasia de endometrio y adenocarcinoma, se decidió en esta primera parte de la investigación presentar el análisis de las características histopatológicas de los casos vistos de hiperplasia de endometrio.

En el estudio anteriormente referenciado de Uribe y Meza (36), la edad más común de presentación

Tabla IV

INCIDENCIA Y EDAD PROMEDIO EN EL DIAGNÓSTICO DE HIPERPLASIA ENDOMETRIAL

\begin{tabular}{|c|c|c|c|c|c|c|}
\hline & & \multicolumn{2}{|c|}{$\begin{array}{l}\text { Hiperplasia endometrial } \\
\qquad \text { sin atipia }\end{array}$} & \multicolumn{2}{|c|}{$\begin{array}{l}\text { Hiperplasia endometrial } \\
\text { con atipia }\end{array}$} & \multirow[t]{2}{*}{ Total } \\
\hline & & $\begin{array}{l}\text { Simple sin } \\
\text { atipia }\end{array}$ & $\begin{array}{l}\text { Compleja sin } \\
\text { atipia }\end{array}$ & $\begin{array}{l}\text { Simple con } \\
\text { atipia }\end{array}$ & $\begin{array}{l}\text { Compleja con } \\
\text { atipia }\end{array}$ & \\
\hline Biopsias & $\mathrm{n}$ & 115 & 20 & 7 & 26 & 168 \\
\hline endometrio & $\%$ & 68,5 & 11,9 & 4,2 & 15,5 & 100 \\
\hline $\begin{array}{l}\text { Edad promedio } \\
\text { de diagnostico }\end{array}$ & & 45,5 años & 42,7 años & 44,3 años & 46,7 años & 44,8 años \\
\hline
\end{tabular}


Tabla V

HIPERPLASIA ENDOMETRIAL SEGÚN RANGOS DE EDAD, CON O SIN ATIPIAS, SIMPLES O COMPLEJAS

\begin{tabular}{|c|c|c|c|c|c|c|c|c|c|c|}
\hline \multirow{3}{*}{$\begin{array}{l}\text { Rango edad al } \\
\text { diagnóstico } \\
\text { (años) }\end{array}$} & \multicolumn{4}{|c|}{$\begin{array}{l}\text { Hiperplasia endometrial } \\
\qquad \sin \text { atipia }\end{array}$} & \multicolumn{4}{|c|}{$\begin{array}{l}\text { Hiperplasia endometrial } \\
\text { con atipia }\end{array}$} & \multicolumn{2}{|c|}{ Total } \\
\hline & \multicolumn{2}{|c|}{$\begin{array}{l}\text { Simple sin } \\
\text { atipia }\end{array}$} & \multicolumn{2}{|c|}{$\begin{array}{l}\text { Compleja sin } \\
\text { atipia }\end{array}$} & \multicolumn{2}{|c|}{$\begin{array}{l}\text { Simple con } \\
\text { atipia }\end{array}$} & \multicolumn{2}{|c|}{$\begin{array}{l}\text { Compleja con } \\
\text { atipia }\end{array}$} & \multirow[b]{2}{*}{$\mathrm{n}$} & \multirow[b]{2}{*}{$\%$} \\
\hline & $\mathrm{n}$ & $\%$ & $\mathrm{n}$ & $\%$ & $n$ & $\%$ & $\mathrm{n}$ & $\%$ & & \\
\hline$\leq 30$ & 4 & 2,4 & 2 & 1,2 & 0 & 0 & 0 & 0 & 6 & 3,6 \\
\hline $31-40$ & 20 & 11,9 & 4 & 2,4 & 1 & 0,6 & 4 & 2,4 & 29 & 17,3 \\
\hline $41-50$ & 68 & 40,5 & 13 & 7,8 & 5 & 2,9 & 17 & 10,1 & 103 & 61,3 \\
\hline $51-60$ & 20 & 11,9 & 1 & 0,6 & 1 & 0,6 & 5 & 2,9 & 27 & 16,1 \\
\hline$\geq 60$ & 3 & 1,8 & 0 & 0 & 0 & 0 & 0 & 0 & 3 & 1,8 \\
\hline TOTAL & 115 & 68,5 & 20 & 11,9 & 7 & 4,2 & 26 & 15,5 & 168 & 100 \\
\hline
\end{tabular}

del adenocarcinoma de endometrio estuvo entre los 55 y 59 años (36) y en nuestras pacientes el pico de presentación de la hiperplasia de endometrio fue a los 44,8 años es decir unos 10 años antes, lo cual está de acuerdo con lo descrito en la literatura mundial $(8,9,15,18,19,33,34)$. Llama la atención que no hubo diferencia en cuanto a promedio de edad en los diferentes cuatro subtipos de hiperplasia endometrial (Tabla IV).

Como patólogos consideramos que la clasificación que usamos (Tabla I), en la que fundamentalmente se tiene en cuenta dos criterios morfológicos grandes, uno arquitectural que divide las lesiones en simples o complejas, y otro citomorfológico, que las categoriza como atípicas o no atípicas, es que es altamente reproducible y ha permitido estudiar y conocer el curso natural de este espectro de lesiones, el cual fue propuesto por primera vez por Kurman y cols (7), quién en 1985 presentó 170 pacientes de las cuales el $54 \%$ eran simples sin atipias, $17 \%$ complejas sin atipias, $7,6 \%$ simples con atipia y finalmente el $20,4 \%$ compleja con atipia, lo cual se traduce en un total de $28 \%$ de pacientes con atipia; porcentaje mucho más alto que el por nosotros encontrado $(19,7 \%)$ en una serie con similar número de casos, pero significativamente menor que el encontrado por Horn y cols (8), la cual fue de $64,1 \%$ en una serie de 39 pacientes. El porcentaje más bajo al respecto es el de Huang y cols (10) con solo 2 casos, equivalentes al $3,4 \%$ de un total de 58 pacientes.

Como era de esperarse el mayor número de pacientes vistos estuvo en el grupo de la hiperplasia simple sin atipia (115 casos equivalentes al $68 \%$ ) y el menor número de pacientes fueron el subgrupo de la hiperplasia simple con atipia (7 pacientes equivalentes al $4,2 \%$ ); categoría que para algunos expertos no existe (10). Los casos de hiperplasia compleja con o sin atipia aunque en menor porcentaje no difirieron sustancialmente del reportado en el trabajo clásico de Kurman y cols (7).

\section{BIBLIOGRAFÍA}

1. Kurman RJ, Norris HJ. Endometrial hyperplasia and related cellular changes. In: Blaustein's Pathology of the Female Genital Tract, 4th ed, Kurman, RJ (Ed), New York: Springer-Verlag, 1994. p. 441.

2. Weiderpass E, Adami HO, Baron JA, Magnusson C, Bergström R, Lindgren A, Correia N, Persson I. Risk of endometrial cancer following estrogen replacement with and without progestins. J Natl Cancer Inst 1999;91:1131-7.

3. Silverberg SG, Kurman RJ. Tumors of the uterine corpus and gestational trophoblastic disease. 1992; Atlas of tumor pathology. Washington DC:Armed Forces Institute of Pathology.

4. Ronnett BM, Kurman RJ. Precursor lesions of endometrial carcinoma. In: Kurman RJ, ed. Blaustein's Pathology of the Female Genital Tract, 5th ed. New York: Springer-Verlag, 2002:467-500.

5. Kummar V, Abbas A, Fausto N. Robbins and Cotran Pathologic Basis of Disease, 7ed. Elsevier 2004; 22:1088-108.

6. Tavassoeli FA, Devilee P. IARC WHO Classification of Tumors. Pathology and genetics. Tumours of the breast and female genital organs. 3ed. 2003; 4: 228-30.

7. Kurman RJ, Kaminski PF, Norris HJ. The behaviour of endometrial hyperplasia. A long-term study of 'untreated' hyperplasia in 170 patients. Cancer 1985;56:403-12.

8. Horn LC, Schnurrbusch U, Bilek K, Hentschel B, Einenkel J. Risk of progression in complex and atypical endometrial hyperplasia: clinicopathologic analysis in cases with and without progestogen treatment. Int J Gynecol Cancer 2004;14:348-53.

9. Horn LC, Meinel A, Handzel Romy, Einenkel J. Histopathology of endometrial hyperplasia and endometrial carcinoma: An update. Ann Diagn Pathol 2007;11:297-311. 
10. Silverberg SG. Problems in the differential diagnosis of endometrial hyperplasia and carcinoma. Mod Pathol 2000;13:309-27.

11. Montgomery BE, Daum GS, Dunton CJ. Endometrial Hyperplasia: A Review. Obstet Gynecol Survey 2004;59(5):368-75.

12. Martínez PL, Atero MD, Bosch JM, et al. ¿Hiperplasia endometrial atípica o adenocarcinoma de endometrio? Un reto. Prog Obstet Ginecol 2008;51(10):605-9.

13. Bergeron C, Nogales FF, Masseroli M, Abeler V, Duvillard $\mathrm{P}$, Muller-Holzner $\mathrm{E}$, Pickartz $\mathrm{H}$, Wells $\mathrm{M}$. A multicentric European study testing the reproducibility of the WHO classification of endometrial hyperplasia with a proposal of a simplified working classification for biopsy and curettage specimens. Am J Surg Pathol 1999;23:1102-8.

14. Mutter GL. Endometrial intraepithelial neoplasia (EIN): will it bring order to chaos? The Endometrial Collaborative Group. Gynecol Oncol 2000;76:287-90.

15. Hecht J, Ince TA, Baak JP, Baker HE, Ogden MW, Mutter GL. Prediction of endometrial carcinoma by subjentive endometrial intraepitelial neoplasia diagnosis. Mod Pathol 2005;18:324-30.

16. Sansal I, Sellers W. The Biology and Clinical Relevance of the PTEN tumor Suppressor Pathway. J Clin Oncol 2004;22:2954-63.

17. Baak JP, Mutter GL, Robboy S, van Diest PJ, Uyterlinde AM, Orbo A, Palazzo J, Fiane B, Løvslett K, Burger C, Voorhorst F, Verheijen RH.. The molecular genetics and morphometry-based endometrial intraepithelial neoplasia classification system predicts disease progression in endometrial hyperplasia more accurately than the 1994 World Health Organization classification system. Cancer 2005;103:2304-12.

18. Baanders-van Halewyn EA, Blankenstein MA, Thijssen $\mathrm{JH}$, de Ridder CM, de Waard F. A comparative study of risk factors for hyperplasia and cancer of the endometrium. Eur J Cancer Prev 1996;5:105-12.

19. Fernández J, Bernet E, Cano R. Lesiones precursoras del carcinoma endometrial. Clin Invest Ginecol Obstet 1999; 26:80-7.

20. The Writing Group for the PEPI Trial. Effects of hormone replacement therapy on endometrial histology in postmenopausal women. The Postmenopausal Estrogen/Progestin Interventions (PEPI) Trial. JAMA 1996; 275:370-5.

21. Wang S, Pudney J, Song J, Mor G, Schwartz PE, Zheng W. Mechanisms involved in the evolution of progestin resistance in human endometrial hyperplasia-precursor of endometrial cancer. Gynecol Oncol 2003;88:108-17.

22. Weiderpass E, Adami HO, Baron JA, Magnusson C, Bergström R, Lindgren A, Correia N, Persson I. Risk of endometrial cancer following estrogen replacement with and without progestins. J Natl Cancer Inst 1999;91:1131-7.

23. Unfer V, Casini ML, Costabile L, Mignosa M, Gerli S, Di Renzo GC. Endometrial effects of long-term treatment with phytoestrogens: a randomized, double-bind, placebo-controlled study. Fertil Steril 2004;82:256-7.

24. Petridou E, Belechri M, Dessypris N, Koukoulomatis $P$, Diakomanolis $E$, Spanos $E$, et al. Leptin and body mass index in relation to endometrial cancer risk. Ann Nutrit Metabol 2002;46:147-51.

25. Carreras R, Mancebo G. Obesidad y Cáncer de Endometrio. Med Clin (Barc) 2007;128(5):176-7.

26. Ricci E, Moroni S, Parazzini F, Surace M, Benzi G, Salerio B, Polverino G, La Vecchia C. Risk factors for endometrial hyperplasia: Results from a case-control study. Int J Gynecol Cancer 2002; 12: 257-60.

27. Maxwell GL, Risinger JI, Gumbs C, Shaw H, Bentley RC, Barrett JC, Berchuck A, Futreal PA. Mutation of the PTEN tumor suppressor gene in endometrial hyperplasias. Cancer Res 1998;58:2500-3.

28. Mutter GL, Lin MC, Fitzgerald JT, Kum JB, Baak JP, Lees JA, Weng LP, Eng C. Altered PTEN expression as a diagnostic marker for the earliest endometrial precancers. J Natl Cancer Inst 2000; 92:924-30.

29. Niemann TH, Trgovac TL, McGaughy VR, VaccareIlo L. bcl-2 expression in endometrial hyperplasia and carcinoma. Gynecol Oncol 1996;63:318-22.

30. Yoshinaga K, Sasano H, Furukawa T, Yamakawa $H$, Yuki M, Sato S, Yajima A, Horii A. The PTEN, BAX, and IGFIIR genes are mutated in endometrial atypical hyperplasia. Jpn J Cancer Res 1998;89:985-90.

31. Leslie NR, Downes CP. PTEN: the down side of PI 3- kinase signalling. Cell Signal 2002;14:285-95.

32. Maruo T, Laoag-Fernandez JB, Pakarinen P. Effects of the levonorgestrel-releasing intrauterine system on proliferation and apoptosis in the endometrium. Hum Reprod 2001;16:2103-8.

33. Sherman ME. Theories of endometrial carcinogenesis: a multidisciplinary approach. Mod Pathol 2000;13:295308.

34. Trimble CL, Kauderer J, Zaino R, Silverberg S, Lim $\mathrm{PC}$, Burke J, et al. Concurrent endometrial carcinoma in women with a biopsy diagnosis of atypical endometrial hiperplasia. Cancer. 2006;106:812-9.

35. Lidor A, Ismajovich B, Confino E, David MP. Histopathologic findings in 226 women with postmenopausal uterine bleeding. Acta Obstet Gynecol Scand 1986;65:41-3.

36. Uribe CJ, Meza EE. Incidencia de cáncer en el Área Metropolitana de Bucaramanga, 200-2004. MedUNAB 2007;10(3):147-72. 